\title{
THE ASSOCIATION OF GENERALIZED ARTERIOLAR SCLEROSIS WITH HIGH BLOOD PRESSURE AND CARDIAC HYPERTROPHY IN CHRONIC NEPHRITIS
}

\author{
BY ARNOLD BRANCH AND GEOFFRY C. LINDER \\ (From the Hospital of the Rockefeller Institute for Medical Research, New York)
}

(Received for publication June 25, 1926)

In the present paper we report observations on the microscopic examination of the vessels of the kidney and other organs in 10 consecutive cases of chronic nephritis in young patients coming to autopsy after previous clinical observation and functional study for some time in the hospital. The data may assist in the eventual clarification of the relationships of renal function and blood pressure changes to cardiac and vascular alterations.

\section{DEFINITIONS OF PRONOUNCED HYPERTENSION AND OF CHRONIC HYPERTROPHY}

The choice of an arbitrary standard by which to decide the presence or absence of pronounced hypertension is a matter of some difficulty, especially since the course of the case in hospital often fails to give a true representation of the whole course of the disease. Fall in blood pressures, (especially systolic pressures) frequently occurs in the terminal stages, even when not attributable to cardiac failure and terminal infection. It has seemed best to use a double criterion, and to class cases as having pronounced hypertension when the systolic pressure has been usually above 180 , or when the diastolic pressure has been usually above $110 \mathrm{~mm}$. of mercury.

Normal limits of the heart weight are even more difficult to establish than the limits of blood pressure. Heart weights vary with body weight, sex, age, and nutrition of the individual. We have charted the figures of absolute heart weights as given by Müller (1) for males and females of various body weights and in different age periods, excluding cases dying from diseases which are frequently associated 
with cardiac hypertrophy, e.g., endocarditis, pericarditis, myocarditis, nephritis, endarteritis and aneurysm, cerebral hemorrhage, chronic pneumonia, pulmonary emphysema, and polyarthritis. We insert one such chart of "normals" as chart 1 , the particular chart being the heart weights of males of 50 to $55 \mathrm{kgm}$. body weight. It is obvious that although the mean of the absolute heart weight remains more or

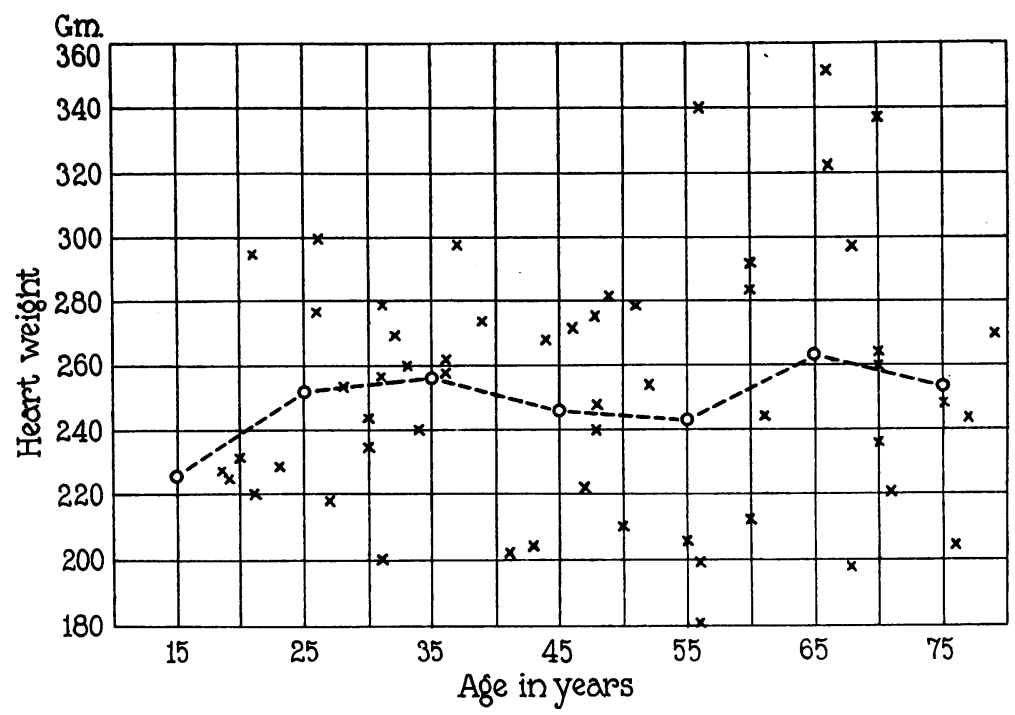

Chart 1. Graphic Representation of Muller's Figures of Absolute Heart Weights of "Normal" Males of 50-55 kgm. Body Weight

$X=$ individual absolute heart weights.

$0=$ mean absolute heart weight in each given age period.

less constant in adults of varying ages above 25, the variation of individual observations in each age period is great. From a study of similar charts of males and females of different body weights we concluded that the values, 400 grams for females and 450 grams for males, indicate the upper extremes of normal gross heart weights for individuals of average size, such as were the seven adults in the series of cases reported below. These maxima of gross heart weights are nearly double the average of absolute heart weights observed by Müller. Weights exceeding them may be accepted as indicating 
definite hypertrophy; but a weight considerably lower by no means excludes the existence of heart enlargement in the individual.

\section{THE ANATOMICAL LESIONS OF ARTERIOLAR SCLEROSIS}

Sclerosis of the small vessels, first described by Gull and Sutton (2) as "arterio-capillary fibrosis," was later studied and differentiated from sclerosis of the larger vessels by Jores (3), and has more recently received extensive investigation by Evans (4) who proposed for it the term "diffuse hyperplastic sclerosis."

Anatomically, according to Evans, the lesions differ somewhat with the size of the vessel. In the smallest arterioles, such as the "afferent arterioles" of the glomeruli, the lesions consist of proliferation of the endothelium of the intima, with hyaline degeneration of the intima, which afterwards frequently shows fatty changes. Narrowing or obliteration of the lumen of the vessel is brought about by these changes. Medial hyperplasia and occasionally perivascular adventitial fibrosis occur. In the vessels from which these smallest arterioles spring, that is in the so-called interlobular arteries of the kidney, those branches of the splenic vessels which run along with the fibrous trabeculae, and in other arterioles of like size, the lesion begins somewhat differently. The first change observed is hyperplasia of the internal elastic membrane, with thickening of the membrane and lamination of the elastic fibres. Hyaline and occasionally fatty changes are to be observed. The lesions occur in the kidney, spleen, brain, retina, liver, pancreas, and occasionally in the appendix. The skeletal muscles and heart are singularly free.

Our observations of these arteriolar lesions accord with Evans' description. In accord with Evans and with Fishberg (5) we have found the lesion most frequently in kidney, spleen, and pancreas, in the order given.

\section{RESUMÉ OF CASE HISTORIES AND AUTOPSY FINDINGS}

\section{(For details see chart 2 and appended protocols)}

Cases 1 and 2. Children of 3 years with acute glomerulonephritis passing into chronic glomerulonephritis of the nephrotic edematous type with the nephrotic element predominant. Patients died of intercurrent infections at this stage of the disease. Renal function was still fairly good, as indicated by the blood urea, although probably 


\section{Chart 2. Summary of the Cimntcal and Pathologi}

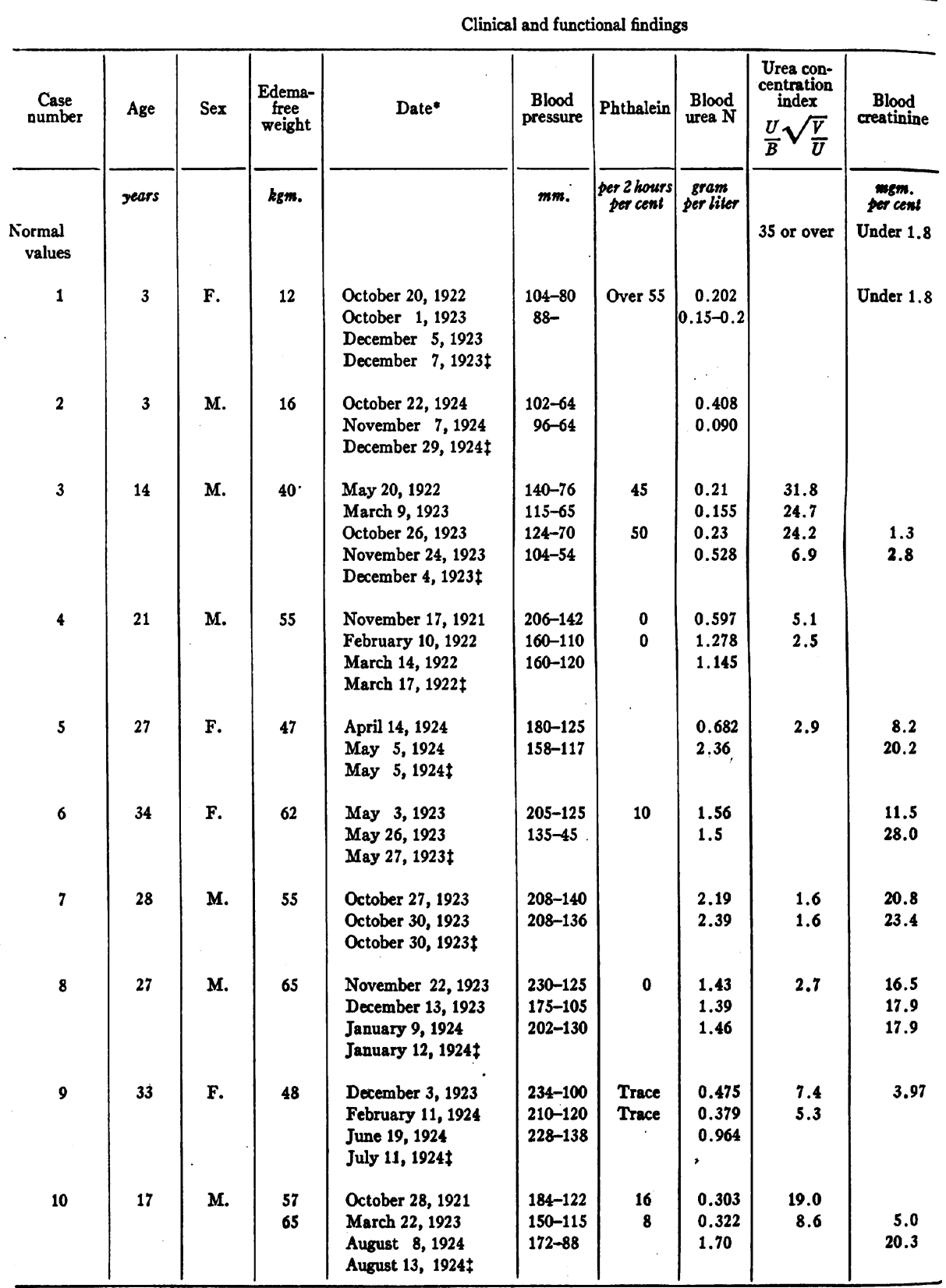

- Dates given are those of phthalein, blood urea $\mathrm{N}$ and creatinine determination. Other data in the same line were obtained within 5 days of date given.

† Figures so marked represent plasma $\mathrm{CO}_{2}$ capacity, expressed in millimols.

$\ddagger$ Date of death. 


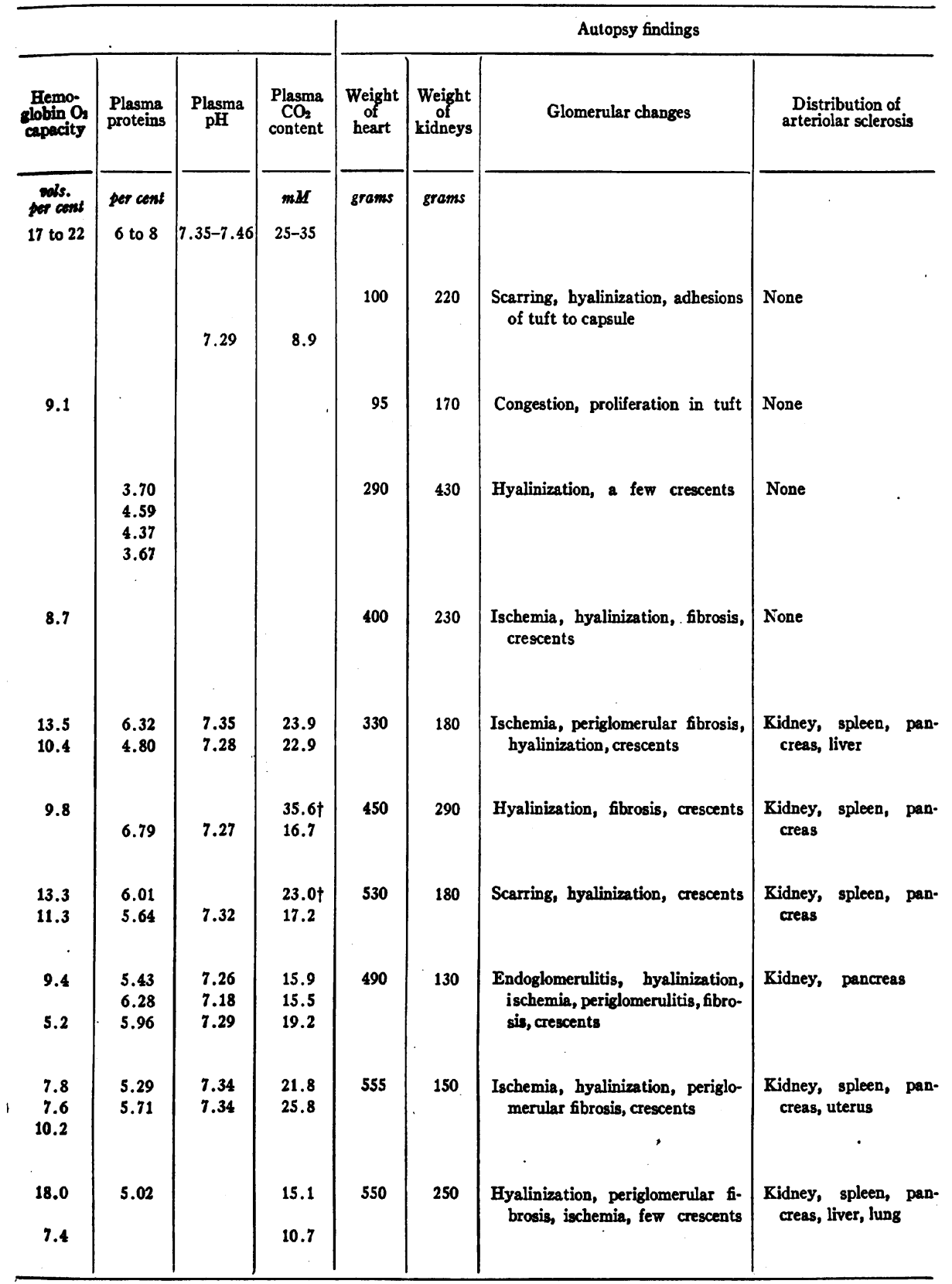


below normal. (Unfortunately the difficulty of obtaining from infants urine samples excreted over definite periods prevented the determination of urea concentration indices.) Blood pressures were normal. Arterioles were normal. Heart weights were probably normal for the age of the patients.

Case 3. Boy of 14. Acute glomerulonephritis progressing into chronic glomerulonephritis of edmatous type with gradual loss of urea concentrating power. Death by intercurrent infection intervened before the functional loss itself reached a fatal degree. Blood pressure was normal throughout. Arterioles and heart weight were normal at autopsy.

Case 4. Young adult, 21. Chronic glomerulonephritis with edema, ending in uremia about 9 months after first symptoms. Renal function was low from the first observation. Blood pressure was definitely above normal (160-206 systolic, 110-140 diastolic) throughout observed course. Arteries in fundi were tortuous and narrowed, but at autopsy arterioles were normal in the kidneys. Heart weight, 400 grams, was within normal maximum.

Cases 5, 6, 7, 8, 9, and 10. All young adults with typical advanced, malignant glomerulonephritis, with great loss of urea concentrating power and increase of blood urea concentration. All had advanced changes in the fundi. All had high blood pressure. All died of uremia except case 8 , in which cardiac failure caused exitus. At post mortem examination all showed arteriolar sclerosis of kidneys, spleen and pancreas, but not of the muscles. All had enlarged hearts except case 5, whose gross heart weight was 330 grams. This case progressed very rapidly to death and the arteriolar lesion was of an acute type.

\section{DISCUSSION}

The frequent concurrence of chronic nephritis, hypertension, cardiac enlargement, and arteriolar sclerosis has long been noticed, and has raised discussion concerning the order of incidence and causal relationships of these respective changes. However, the difficulty of obtaining evidence even of the order of incidence of the anatomical changes is exemplified by the autopsies on all our patients (nos. 4 to 10) who came 
to nephritic deaths. Each patient had hypertension and diffuse glomerular lesions, and all but one had cardiac enlargement. All but one case (no. 4) showed also arteriolar sclerosis. By the time nephritis has run its course all these conditions are usually present. It is only by opportunities to examine material from cases of chronic nephritis that have died of intercurrent causes that one is likely to obtain information from which deductions as to the order of incidence can be drawn. Even when such deductions are possible, they permit only inferences concerning the causal relationships. For example, if it were found that hypertension invariably precedes arteriolar sclerosis in nephritis, it would still be uncertain whether the hypertension causes the sclerosis, or whether both are independent results of the nephritis, one developing more rapidly than the other. Our case 4 in which there was fatal nephritis with hypertension but no arteriolar sclerosis, shows that the latter does not in all cases precede hypertension, and hence that some condition in the disease other than arteriolar sclerosis can incite increase in blood pressure. The histories of all these cases are free from evidence of hypertension preceding the nephritis, although it is conceivable that hypertension without subjective symptoms may have been present. As our data stand the apparent interpretation concerning the order of incidence is nephritis, hypertension, arteriolar sclerosis, in this type of rapidly progressing "malignant" hypertensive nephritis.

Evans (4) has come to the conclusion that glomerulitis and arteriolar sclerosis are two separate and independent results of one cause, probably infection. As to the direct cause of the hypertension usually encountered in advanced chronic glomerulonephritis he makes no suggestion, but argues from the small proportion of the arterioles in the body affected by sclerosis (the muscles being exempt) that mere mechanical resistance to the heart pump by contraction of the affected arterioles would be quite insufficient to produce the increase in pressure. With his conclusion that some factor other than arteriolar sclerosis is responsible for hypertension in nephritis our findings in case 4 are in accord.

Fishberg (5) has found that senile hypertension is invariably associated with arteriolar sclerosis. He believes that the hypertension promotes the sclerosis, which is a normal senile result of physiological wear 
and tear, and is merely accelerated by the added strain of hypertension. Concerning the origin of the hypertension he offers no explanation.

The regular association of senile hypertension and arteriolar disease can hardly indicate which one is responsible or whether both arise from a common cause. When renal insufficiency occurs in an elderly subject with hypertension (as it did in about 7 per cent of Fishberg's cases) the order of incidence appears to be

$$
\left.\begin{array}{l}
\text { arteriolar sclerosis } \\
\text { hypertension }
\end{array}\right\} \rightarrow \text { nephritis }
$$

with priority in question between sclerosis and hypertension.

It appears quite possible, as Volhard and Fahr (6) believed, that in one group of nephritics, typified by the young subjects reported in this paper, the nephritis is the initial lesion, exciting the development of hypertension and arteriolar sclerosis; while in another, usually older group, arteriolar sclerosis is the initial lesion, exciting the hypertension, with renal insufficiency eventually developing either from sclerotic strangulation of the glomeruli or from inflammatory processes added to the sclerotic strangulation. In both groups cardiac enlargement presumably follows hypertension.

The hypertension has been conceived as a physiological effort to restore blood flow and function which have been diminished by lesions in either the glomerules or the arterioles of the kidney. However whether hypertension is such a beneficient compensatory effort, or an injurious malfunction, increasing the ill effects of the disease, appears to be still open to debate. Possibly hypertension is necessary for proper renal function in some cases, but it may be so unduly exaggerated that the strain results in cardiac or cerebral death while the renal function is still adequate to support life. That the organism should distribute burdens so equally that the systems among which they are divided all endure for the same maximum period, would be an unreasonable expectation with respect to the capacity for adjustment.

Likewise concerning the causal relationships of concurrent nephritis, hypertension, and arteriolar sclerosis, hypotheses such as we have sketched above are nothing more at present than incomplete state- 
ments of problems to be solved. Limiting discussion to nephritis of the type reported here (young subjects progressing to chronic, rapidly fatal renal insufficiency), and excluding primary nephrosclerosis with secondary renal insufficiency, the following alternatives appear to be left open for decision from future evidence. For cases of the type considered, in which nephritis is apparently the primary cause of the syndrome, either: (a) Hypertension may next arise and in turn cause arteriolar sclerosis (all of our hypertension cases might fall within this group); or (b) arteriolar sclerosis may occur and induce hypertension ( 6 of our 7 hypertension cases might be thus explained); or (c) hypertension and sclerosis may originate independently as a result of the nephritic toxins. (This would presumably be the case if either hypertension or sclerosis were found frequently without the other.) (d) Finally nephritis may not be the primary cause of the other conditions, but with them the result of a common injurious agent or agents, which incite the development of all three in varying sequence. (This would cover all possible findings, but explain the origin of none, until the injurious agents, infectious, metabolic, or other, were identified.)

It is quite possible that no generalization can be made, but that the order of incidence and the causal relationships vary, even when the terminal results are similar.

SUMMARY

1. This report is based on clinical and anatomical findings in 10 young subjects (under 34) with chronic glomerulonephritis. Three cases died of intercurrent infections, 7 came to typical nephritic death, 6 in uremia, 1 from heart failure.

2. The 3 cases (in children aged 3, 3, and 14 years) which terminated by intercurrent infection. showed relatively mild but definite, diffusely distributed, glomerular lesions. They were free from hypertension, arteriolar sclerosis, or cardiac hypertrophy, and demonstrate that glomerulonephritis can develop to a considerable degree without such results.

3. All 7 cases which progressed to typical nephritic deaths showed hypertension and neuroretinitis, together with cardiac enlargement, gross in 5 cases, moderate but probably definite in 2 . These findings 
accord with the current view that cardiac enlargement in nephritis is the result of hypertension.

4. In 6 of these 7 cases arteriolar sclerosis of the parenchymatous organs was found at autopsy. The concurrence of arteriolar sclerosis so frequently with retinitis and hypertension shows that the presence of hypertension in nephritis indicates usually but not always a co-existing arteriolar sclerosis.

5. The non-conforming case (case 4) confirms Volhard and Fahr $(5$, p. 41$)$ in showing that arteriolar sclerosis may be absent even when neuroretinitis and hypertension have existed for several months, accompanied by renal insufficiency advancing to uremia.

\section{BIBLIOGRAPHY}

1. Müller, Wilhelm: Die Massenverhältnisse des Menschlichen Herzens. Hamburg and Leipsiz, (Voss) 1883.

2. Gull, W., and Sutton, B.: Proc. New Sydenham Soc., 1894, 325.

3. Jores, L.: Wesen und Entwichkelung der Arteriosklerose. Wiesbaden Bergmann, 1903.

4. Evans, G.: Quart. Jour. Med., 1921, xiv, 260. A Contribution to the Study of Arterio-Sclerosis, with Special Reference to its Relation to Chronic Renal Disease.

5. Fishberg, Arthur, M.: Arch. Int. Med., 1925, xxxv, 650. Anatomic Findings in Essential Hypertension.

6. Volhard, F., and Fahr, Th.: Die Brightsche Nierenkrankheit. Berlin, Springer, 1914.

\section{OUTLINE OF CASE HISTORIES AND AUTOPSY FINDINGS'}

Case 1. Hospilal No. 4601. Female, 3 years old. She had suffered from frequent "colds." Loss of appetite and of good nature began in May, 1922, also swelling of face and extremities. The urine was dark and diminished in amount. Nephritis was diagnosed. Ascites became pronounced, and required tapping. After 12 weeks at home she was taken to the Brooklyn Hospital, where repeated tappings were necessary. Eight weeks later she was transferred to this Institute. She was moderately comfortable. There were enlarged tonsils. The abdomen was immensely distended, and there was edema of the legs and sacral region. The

${ }^{1}$ The histories and tabulation of functional data have in part been completed during the absence of both the authors. The verification and completion of data from the hospital records, and the corresponding final version of the text, have been undertaken by Dr. J. F. McIntosh. 
heart was negative. Blood pressure, 102-80. Blood urea and phthalein excretion were normal. The urine contained albumin to the extent of 12 grams per liter. There were granular and hyaline casts, numerous white cells, and very few red cells in the centrifugings. The Wasserman reaction was negative. For 4 months she required frequent abdominal paracentesis. Then reaccumulation of fluid decreased, and she went home in fair condition after a 9 months stay. Two months later she returned, with edema and ascites. These symptoms yielded to treatment, but a streptococcus septicemia proved fatal 15 weeks after the second admission.

Autopsy findings. Body weight, $10 \mathrm{kgm}$. Heart weight, 100 grams, kidneys weighed (horse shoe), 220 grams. The kidney was large, pale, greyish and gelatinous; the surface was smooth and the capsule stripped readily. The cortex was wide and yellowish. The cut surface bulged. Microscopically, there was cystic dilatation of the tubules, the lining epithelium was degenerated and contained fat and doubly refractive bodies. Some interstitial scarring and lymphocytic infiltration were present. The blood vessels were not thickened. Many glomeruli were scarred and hyaline and the tufts were often adherent to Bowman's capsule, but there were no crescents. No arteriolar sclerosis nor cardiac hypertrophy was found. In addition to the pathological changes in the kidney there was streptococcus hemolyticus septicemia with peritonitis, pleurisy, pericarditis and abscess of the cheek. There were also noted fatty degeneration of the aorta and liver, acute splenitis and parenchymatous degeneration of organs.

Case 2. Hospital No. 5102. Male, 3 years. His family history, birth, and development were not of significance. He suffered from a chronic cough, and on two occasions had febrile attacks considered to be tonsillitis. Edema began insidiously two months before admission. Three days before he came to the hospital, there was increase in the edema, and later vomiting, thirst, and malaise. He was febrile on admission. The heart and lungs presented no abnormality. A moderate grade of ascites and peripheral edema were present. Blood pressure was 102-64. The urine showed a specific gravity of 1031; sugar absent; albumin $16 \mathrm{gm}$. per liter (Esbach). Blood urea nitrogen was never dangerously high. In the centrifugings were many hyaline and granular casts, pus, red cells, red cell casts, and epithelial casts.

The blood count showed red blood cells, 4,510,000; white blood cells, 32,000; hemoglobin, 9.1 volumes per cent oxygen capacity. After two weeks stay the edema decreased rapidly, and his progress for the next 4 weeks seemed favorable, except for a persistent leucocytosis. Then came a coryza, followed 10 days later by fever and prostration. Finally an acute colitis and peritonitis ended the picture.

Autospy findings. Body weight, $16 \mathrm{kgm}$. Heart weight, 95 grams. Kidneys weighed 170 grams. The surface of the kidney was smooth, pale, and greyish yellow in color; the capsule was not adherent; the stellate veins were prominent. The cut surface bulged; the cortex was greyish yellow and well differentiated from 
the medulla. The markings were fairly distinct. Microscopically there were no glomerular crescents, but the glomeruli were cellular and somewhat congested. The tubular cells were definitely large, swollen and pale and many contained fat and doubly refracting bodies. There were no vascular changes. Other pathological findings were purulent peritonitis and ulcerative colitis (B.dysenteriae), septicemia, sero-fibrinous pleurisy and focal pneumonia (B. coli communis).

Case 3. Hospital No. 4546. Male, aged 14. His history included removal of adenoids and tonsils 3 years before. Symptoms began with edema a week before admission, which led to the discovery of albuminuria. He presented edema of face, abdominal wall, legs, and sacral region. He was a mouth breather, with a facies of marked "adenoid" type. A little muco-pus was to be seen in the nasopharynx. There was a small amount of pleural effusion. There was no radial sclerosis. The heart showed no abnormality. The blood pressure was 135-90. The urine was smoky, and contained 4 grams of albumin per liter (Esbach). In the deposit were numerous red and white cells, and a fair number of coarsely granular casts. Red blood cells, 4,150,000. White blood cells, 14,000. The Wassermann reaction was negative. The edema gradually subsided, but the hematuria persisted. The anemia grew worse, and the fundi which had been normal on admission developed papilledema, exudate, and a few hemorrhages. He was discharged after 10 months stay. He led a life of limited exertion for 8 months longer, when signs of acute illness supervened. He returned to the hospital, where he died of general septicemia. Hemolytic streptococci were isolated from the blood cultures.

Autopsy findings. Body weight, $38.6 \mathrm{kgm}$. Heart weight, 290 grams. The kidneys weighed 430 grams. They were large, pale, slightly granular, and on section they bulged and the cortex was pale yellow and wide. Microscopically the glomeruli were large and cellular; some showed partial, others complete, hyalinization, but the majority were little affected. A few crescents were found. The tubules were slightly degenerated and some contained fat. Little scarring was noted and only slight infiltration with small round cells. No doubly refracting bodies were present. The vessels were not thickened. Other pathological lesions were Streptococcus hemolyticus septicemia, with peritonitis, pleurisy, pneumonia and pericarditis, fatty degeneration of the aorta and acute splenitis. No cardiac hypertrophy nor arteriolar sclerosis was present.

Case 4. Hospital No. 4421. Male, aged 21. The patient complained of blurred vision and swollen legs. His antecedents were irrelevant. Nocturia had begun 6 months before, and loss of appetite at about the same time. His vision had been blurred for a month, and for 3 weeks palpitation and dyspnea had been present. Edema of the legs was first noted 3 days before admission. His complexion was pale and sallow. One tonsil was enlarged. The chest was negative. The heart extended $4.5 \mathrm{~cm}$. to the right, and $10 \mathrm{~cm}$. to the left of the mid-line. 
The radial vessels showed no sclerosis. Blood pressure 200-140. There was no edema on admission. The urine showed intense albuminuria with cellular, hyaline, and granular casts, with many red and a few white cells. The Wasserman reaction was negative. The fundi showed marked neuro-retinitis, with numerous patches of exudate and superficial hemorrhage. His functional findings showed a terminal condition. He stayed 3 months in the hospital, with some symptomatic improvement. He returned 18 days after his discharge with a severe acidosis, and died 3 days later in a uremic convulsion.

Autopsy finding. Body weight, $63.1 \mathrm{kgm}$. Heart weight, 400 grams. Kidneys weighed 230 grams. These were contracted, granular and pale. Microscopically the glomeruli were anemic and the majority showed extensive changes, hyalinization, fibrosis or crescent formation. There was extensive interstitial fibrosis with distortion and dilatation of tubules which showed degenerating epithelium and contained casts. There were no thickened blood vessels. Fat was present in some tubules. In short, the kidneys showed extensive and active inflammatory changes but the arterioles were unaffected. Other findings besides glomerular nephritis were edema, ascites, hydro-pericardium and lobular pneumonia. No cardiac hypertrophy and no arteriolar sclerosis was present.

Case 5. Hospital No. 5014. Female, aged 27. She complained of headache, vomiting and defective sight. Her personal history included a simultaneous attack of measles and scarlet fever at 10 years, when she was very ill for some weeks. Since childhood she had had repeated sore throats, which were not relieved by a tonsillectomy at the age of 11 years. For 7 years she had had repeated quinsy. Symptoms of an earlier acute nephritis were lacking. She felt well until a month before admission, when morning nausea began, and was followed rapidly by headache, vomiting and failure of vision. For 4 weeks she neglected medical advice, and there was rapid downward progress. She seemed weary and depressed; her face was pale and a little puffy. The throat was scarred and inflamed. Cardiac dullness was increased in area. The peripheral arteries were definitely thickened. Blood pressure was 180-125. There was no edema. The fundi showed narrowed arteries, whose walls appeared thickened and tortuous. The disc margins were obscured with exudate. Stellate areas of degeneration were seen in the macular regions. The left fundus showed many small hemorrhages. Power to concentrate urea was almost nil. The urine showed albumin 2.2 grams per liter. There were hyalo-granular casts, and fairly numerous red and white cells. Red blood cells 3,360,000; white blood cells, 6,800. Wassermann reaction was negative. There was no acidosis on admission but the $\mathrm{pH}$ and plasma $\mathrm{CO}_{2}$ later fell to pathological values. Her clinical condition grew rapidly worse with corresponding laboratory findings, and she died in uremia 24 days after admission.

Autopsy findings. Body weight, $45.7 \mathrm{kgm}$. Heart weight, 330 grams. Kidneys weighed 180 grams. They were small, the capsules stripped with difficulty, the surfaces were pale and finely nodular. On section the cortex appeared very narrow 
and the arteries stood out prominently. Microscopically a considerable amount of fibrous tissue was seen replacing and distorting the tubules which were dilated, filled with fluid and lined by low epithelium. They contained some fat. Lymphocytes were present in the scars. The glomeruli were anemic, many were scarred and surrounded by fibrous tissue, while others showed hyaline replacement. Crescents were easily found. The small vessels showed very extreme and widespread fatty degeneration and thickening and some elastic tissue increase. Arteriolar sclerosis was present also in the spleen, liver and pancreas. Other findings were lobular pneumonia and pulmonary edema.

Case 6. Hospital No. 4772. Female, aged 34. She complained of nausea, vomiting and failing vision. Her father had died of Bright's disease at 40 years of age. At 7 she had had scarlet fever. The first symptoms began 4 months before admission. Nausea, anorexia, and vomiting, asthenia and loss of weight followed. Her kidney disease was then discovered; seven weeks before admission she became bed-ridden, on account of weakness. Her vision had failed. Her blood pressure had been noted as 260 . Vomiting persisted. On admission her complexion was poor, the face puffy. The heart was slightly enlarged. The blood pressure was 230-150. The fundi showed a typical albuminuric retinitis. The urine contained 7 grams of albumin per liter (Esbach) with red, white, and epithelial cells and granular casts. Hemoglobin was 9.8 volume per cent oxygen capacity. The Wassermann reaction was negative. There was an alkalosis on admission, which was probably to be ascribed to her vomiting. This gave place to a compensated acidosis during her stay. There was a low intermittent fever of unexplained origin. Three weeks after her admissiin she developed symptoms of pyelitis and cystitis, and Staphylococcus aureus was cultivated from the urine. She died in uremia 5 days later.

Autopsy findings. Body weight, $62 \mathrm{kgm}$. Heart weight, $450 \mathrm{gram}$. Kidneys weighed 290 grams. They were rather soft; the capsule stripped easily; the surface was dark purplish red, not granular, and showed small abscesses. The cut surface bulged and was of a purplish gray color; the markings were obscured, and abscesses were seen. Microscopically there was a pyelonephritis with multiple abscesses engrafted on a chronic nephritis. The small vessels were thickened and fatty. The glomeruli showed partial or complete hyalinization, some fibrosis and a few crescents. There was some fibrosis and distortion of tubules. Cardiac hypertrophy was present and the lesions of arteriolar sclerosis were found in the kidney, spleen and pancreas, but not in the liver or adrenal. Other findings were staphylococcus septicemia and fibrinous pericarditis.

Case 7. Hospital No. 4829. Male, aged 28. He complained of vomiting and weakness. He had had typhoid fever 3 years before. A year later, he began to have severe occipital headaches, recurring once a fortnight, and shortly after, nocturia became troublesome. About 2 months before admission pallor and loss of weight were noted, and vomiting began. A bronchitis took him to his doctor, 
and albuminuria and hypertension were then discovered. Dyspnea on exertion was noted, and muscular twitchings were present 3 or 4 days before admission. When admitted he was pale and ill. The heart was enlarged, the beat diffuse, the second sound reduplicated. The peripheral arteries were thickened. Blood pressure was 204-140. There was edema of the legs and over the sacrum. The fundi showed the arteries contracted, blurring of the discs, with a few hemorrhages and retinitis pigmentosa. Power to concentrate urea was reduced to 1.6, compared with normal 40 -fold concentrating power. In the urine, albumin was found, 2.5 grams per liter (Esbach). The deposit contained epithelial cells, leucocytes, a few granular casts, and very few red cells. Red blood cells, 4,130,000. Hemoglobin was 13.5 volume per cent oxygen capacity. There was no acidosis. Next day uremic convulsions appeared. Pericardial friction was heard. He died two days later of respiratory failure.

Autopsy findings. Body weight, $55 \mathrm{kgm}$. Heart weight, 530 grams. Kidneys weighed 120 grams. There was arteriosclerosis of the renal arteries. The kidneys were finely granular and reddish white in color. The capsule was adherent; the cortex was narrow and contained small cysts. Microscopically there was thickening with fatty intimal degeneration and elastic tissue increase in the small arterioles. There was marked interstitial scarring. The tubules were dilated and lined with low epithelium. Many scarred and hyaline glomeruli and glomerular crescents were present. Arteriolar sclerosis was found in the kidney, pancreas, and spleen, but not in the heart, liver, adrenals or testicles. The heart was definitely hypertrophied. Further anatomical diagnoses: ascites, hydropericardium, edema, hydrothorax, and infarcts of lung.

Case 8. Hospital No. 4847. Male, aged 27. He complained of headache, vomiting, and weakness. He was overseas with the American Expeditionary Forces for 2 years. He escaped influenza and trench fever, but suffered from a flesh wound. During the last 12 months in Europe he had occasional headaches. In 1920 he was refused life insurance on account of albuminuria. Headaches became more severe 4 months before admission, and were accompanied by anorexia. During his last 6 weeks at home he failed in strength, and 3 weeks before admission vision began to fail. There was excessive thirst and drowsiness. $\mathrm{He}$ seemed well nourished. His face was pale and puffy. The breath was strikingly urinous. The heart did not seem enlarged. Blood pressure was 230-125. The fundi showed blurring of the discs, with many patches of exudate and some hemorrhages. There was albuminuria to the amount of 2 grams per liter, and the sediment contained many red cells, some white cells, and a few hyaline and granular casts. Power to concentrate urea was reduced to 2 compared with normal 40 -fold concentrating power. The blood showed: red blood cells, 3,168,000; white blood cells, 14,800; hemoglobin, 9.5 volumes oxygen per cent oxygen capacity. The Wassermann reaction was negative. There was a marked acidosis, which was combatted with sodium bicarbonate in five-gram doses thrice daily. This treatment relieved the acidosis, and for the first 4 or 5 weeks there was some symp- 
tomatic improvement. Then he sank gradually, twitchings developed and he died in uremia.

Autopsy findings. Body weight, $65 \mathrm{kgms}$. Heart weight, 490 grams. Kidneys weighed 130 grams. The capsule was slightly adherent, the surface was very pale and slightly granular. The cut surface showed poor differentiation of the cortex and medulla, and the cortical substance was slightly narrowed, pale and gelatinous. Microscopically there was very extensive scarring by dense hyaline and vascular fibrous tissue which was infiltrated with lymphocytes, the kidney substance being relatively inconspicuous. Normal glomeruli were sparse; some showed crescents and endoglomerulitis with fluid in Bowman's space. The majority were the seat of partial or complete hyalinization with periglomerular fibrosis, the tissue being arranged in concentric layers. The tufts were avascular. The tubules were cut off into islands by fibrous tissue and were dilated and lined with low epithelium. They contained serous and hyaline material and cellular casts. The small blood vessels showed thickening of the walls, narrowing of their lumina and fatty degeneration of the intima with lamination and increase in the elastic tissue fibres. Arteriolar sclerosis was found in the pancreas. Other findings were hypertrophy of the heart, ascites, and hydrothorax, pulmonary infarction, and edema.

Case 9. Hospital No. 4863. Female, aged 33. She complained of shortness of breath, cough, and failing vision. She had had adenoids and had suffered from sore throats in childhood. She had borne four children. In January, 1921, she had noted swelling of her feet, and her physician diagnosed kidney trouble. Headache and palpitation followed, and caused her to spend July and August 1922, in the hospital. She did well for a year, though the same symptoms were sometimes present. In October, 1923, her dyspnea was worse and accompanied by a cough. There was orthopnea and nocturia. Her eyesight became impaired 7 weeks before her admission on December 1, 1923. Temperature was $102.6^{\circ} \mathrm{F}$.; pulse, 116; respirations, 28. She could not lie down. There were signs of fluid in both chest cavities, and crepitations throughout the lung areas. The area of cardiac dullness was increased and a faint systolic murmur was heard, and at the base a gallop rhythm. There was a good deal of thickening of the radials. The pulse was regular. The abdomen showed nothing abnormal. There was moderate edema of the feet, legs, and sacro-lumbar region. The eye-grounds revealed tortuous arteries, with exudate here and there. There was no retinal hemorrhage. The urine contained albumin, up to 4 grams per liter and an abundance of hyaline and granular casts. Urea concentrating power was only 7 -fold, compared with normal 40-fold. A moderate number of white cells and a few red cells were seen. Red blood cells 2,368,000; white blood cells, 8,650; hemoglobin, 7.8 volumes per cent oxygen capacity. Her heart responded to treatment and she went home after 7 weeks, feeling much improved. She returned on June 17, with a recurrence of cardiac decompensation, and died of heart failure with terminal pneumonia on July 11, 1924. 
Autopsy findings. Body weight, $51.5 \mathrm{kgm}$. Heart weight, 555 grams. Kidneys weighed 150 grams. The capsule stripped with difficulty. The surfaces were pale, grayish yellow, streaked with fine vessels and finely granular. The cut surface was very pale, the cortical substance narrow, the glomeruli stood out as fine points. One small cyst was seen in the left kidney and a depressed infarct in the right. The differentiation of cortex and medulla was obscured. Microscopically there were local areas of scarring, causing puckering of the surface. These scars were infiltrated with small round cells. The glomeruli were anemic and many had undergone complete or partial hyaline replacement. Others were surrounded by concentric layers of fibrous tissue. Crescents were readily found. The tubules were mostly lined with low epithelium and contained casts. They were not dilated. The small vessels were greatly thickened and contained fat in the intima. Fat was also present in some tubules. Arteriolar sclerosis was found in the pancreas, spleen, and uterus. Other findings were cardiac hypertrophy, atheroma of aorta, fluid in all serous cavities, anasarca, lobular pneumonia, brown induration of lung, and healed gastric ulcers.

Case 10. Hospital No. 4410. Male, aged 17. His history disclosed no etiology for his nephritis. The onset was marked by dyspnea, with swelling of his legs and face. A few days later he went to the hospital where acute Bright's disease was diagnosed. His edema disappeared, but a recurrence with cardiac irregularity brought him to this institution. There was general anasarca, and fluid in the abdominal and in both pleural cavities. Nodal rhythm was present. Blood pressure, 184-136. Retinoscopy showed marked papilledema, and later exudative retinitis, with slight hemorrhage. He was anemic. The urine showed much albumin, with a good many red and white cells, many hyaline and granular casts, and occasional blood casts. There was a marked uncompensated alkali deficit. Convulsions developed. Improvement was slow, but he was able to go home 11 months later. He returned frequently for examination, and a tendency to edema was always present, together with anemia. He died 3 years and 2 months after the onset of his symptoms.

Autopsy findings. Body weight $66 . \mathrm{kgm}$. Heart weighed 550 grams. Kidneys weighed 250 grams. The capsule stripped with slight difficulty exposing a pale, finely granular surface, streaked with fine vascular twigs. On section the cut surface was pale, the cortex and medulla were fairly well differentiated; the cortex was narrow and pale yellow and the glomerular markings were evident. Microscopically the kidney showed extensive scarring. The glomeruli were extensively involved, showing partial or complete hyaline transformation and periglomerular fibrosis. Others were anemic. Crescents were sparse. The tubules were degenerated in places and in others lined with low epithelium. There was evidence of regeneration. A few of the smaller vessels showed fatty degeneration and thickening. Arteriolar sclerosis was found in the pancreas, spleen, liver and pleura as well as in the kidney. Other findings were cardiac hypertrophy, fatty degeneration of the heart, lobular pneumonia, atherosclerosis, calcareous mesenteric lymph glands, ascites, hydrothorax and hydropericardium. 


\section{EXPLANATION OF FIGURES}

\section{Plate 1}

Fig. 1. Case 9. Arteriole lesion of Diffuse Hyperplastic Sclerosis in THE KIDNEY

Frozen section stained with Scharlach $\mathbf{R}$ and hematoxylin. Camera lucida painting $\times 360$.

Fig. 2. Case 8. Artertole Lesion of Diffuse Hyperplastic Sclerosis in THE PANCREas

Frozen section stained with Scharlach $\mathrm{R}$ and hematoxylin. Camera lucida painting $\times 360$.

Fig. 3. Case 5. Arteriole in the Kidney

Stained by Weigert's Elastic Tissue Method. Camera lucida painting $\times 360$.

Plate 2

Fig. 4. Case - Three Arterioles in the Medulla of the Kmney

A. Endothelial proliferation. B and C. Narrowing of lumen due to thickening of wall. Microphotograph $\times 300$.

Fig. 5. Case 4. A Glomerulus Showing a Typical Crescent $(P)$ Which We Consider Diagnostic of Glomerulonephritis

Microphotograph $\times 250$ 


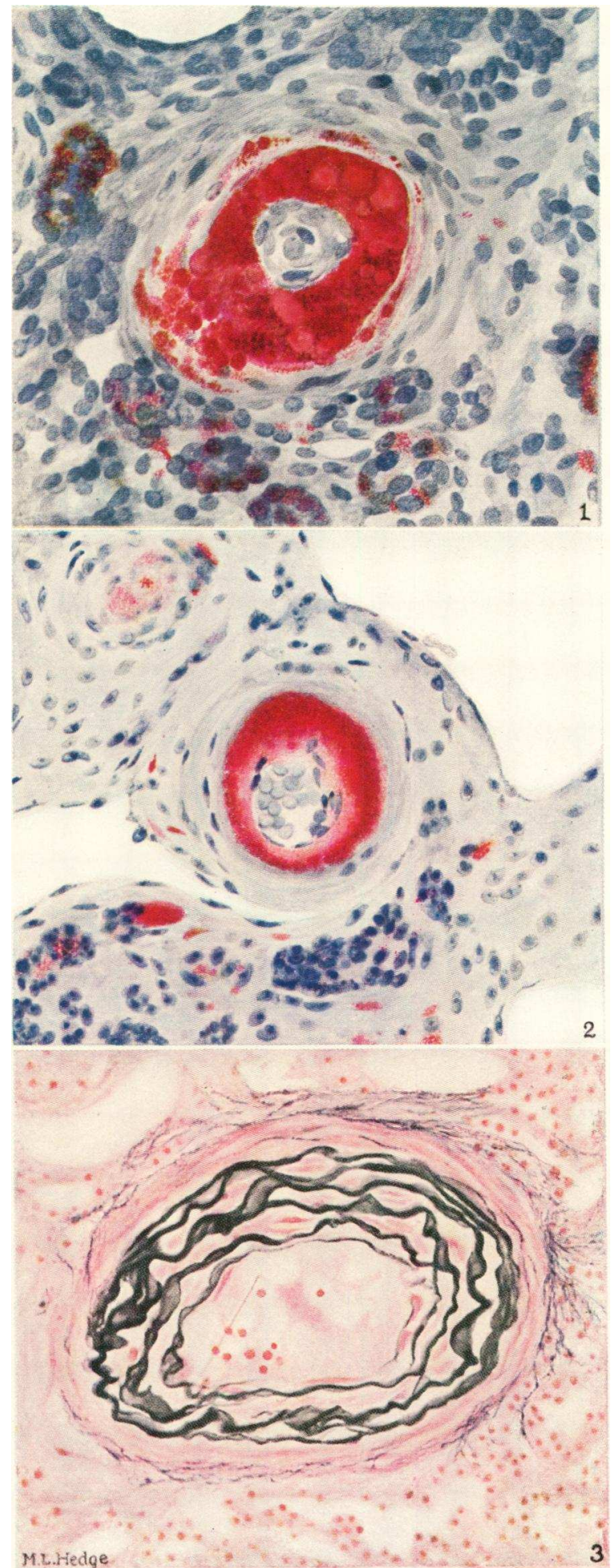

(Branch and Linder: Chronic nephritis.) 


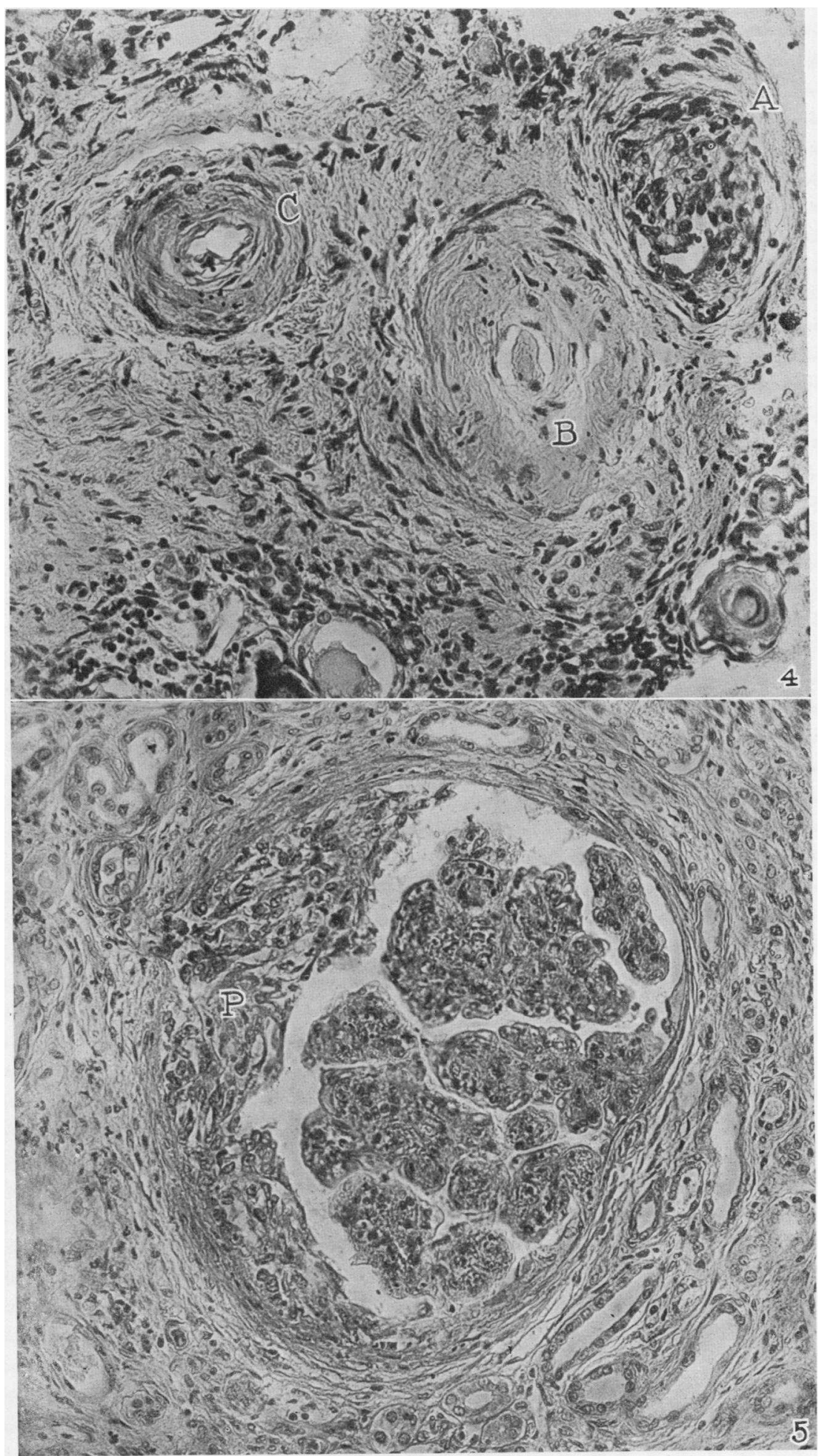

(Branch and Linder: Chronic nephritis.) 